\title{
Reconfigurable 3D plasmonic metamolecules
}

\author{
Anton Kuzyk ${ }^{1 \star}$, Robert Schreiber ${ }^{2 \uparrow}$, Hui Zhang ${ }^{3}$, Alexander O. Govorov ${ }^{3}$, Tim LiedI $^{2}$ and Na Liu ${ }^{1 \star}$
}

A reconfigurable plasmonic nanosystem combines an active plasmonic structure with a regulated physical or chemical control input. There have been considerable efforts on integration of plasmonic nanostructures with active platforms using topdown techniques. The active media include phase-transition materials, graphene, liquid crystals and carrier-modulated semiconductors, which can respond to thermal', electrical ${ }^{2}$ and optical stimuli ${ }^{3-5}$. However, these plasmonic nanostructures are often restricted to two-dimensional substrates, showing desired optical response only along specific excitation directions. Alternatively, bottom-up techniques offer a new pathway to impart reconfigurability and functionality to passive systems. In particular, DNA has proven to be one of the most versatile and robust building blocks ${ }^{6-9}$ for construction of complex three-dimensional architectures with high fidelity ${ }^{10-14}$. Here we show the creation of reconfigurable three-dimensional plasmonic metamolecules, which execute DNA-regulated conformational changes at the nanoscale. DNA serves as both a construction material to organize plasmonic nanoparticles in three dimensions, as well as fuel for driving the metamolecules to distinct conformational states. Simultaneously, the threedimensional plasmonic metamolecules can work as optical reporters, which transduce their conformational changes in situ into circular dichroism changes in the visible wavelength range.

Circular dichroism (CD), that is, differential absorption of left- and right-handed circularly polarized light, of natural chiral macromolecules is highly sensitive to their three-dimensional (3D) conformations ${ }^{15}$. Taking a similar strategy, we create 3D reconfigurable plasmonic chiral metamolecules ${ }^{4,16}$, whose conformation changes are highly correlated with their pronounced and distinct $\mathrm{CD}$ spectral changes in the visible wavelength range. Figure 1a shows the design schematic. Two gold nanorods (AuNRs) are hosted on a reconfigurable DNA origami template ${ }^{7,10}$, which consists of two 14-helix bundles $(80 \mathrm{~nm} \times 16 \mathrm{~nm} \times 8 \mathrm{~nm})$ folded from a long single-stranded DNA (ssDNA) scaffold with the help of hundreds of staple strands ${ }^{13}$. The two origami bundles are linked together by the scaffold strand passing twice between them at one point. To ensure the mobility of the DNA bundles and avoid the formation of a Holliday junction ${ }^{17}, 8$ unpaired bases are introduced to each ssDNA connector (Supplementary Note 1). Twelve binding sites are extended from each origami bundle for robust assembly of one AuNR $(38 \mathrm{~nm} \times 10 \mathrm{~nm})$ functionalized with complementary DNA (Supplementary Note 2). The surface to surface distance of the two AuNRs is roughly $25 \mathrm{~nm}$. Owing to close proximity, the excited plasmons in the two AuNRs can be strongly coupled ${ }^{18}$. The two crossed AuNRs constitute a 3D plasmonic chiral object ${ }^{19-22}$, which generates a theme of handedness when interacting with left- and right-handed circularly polarized light, giving rise to strong CD.
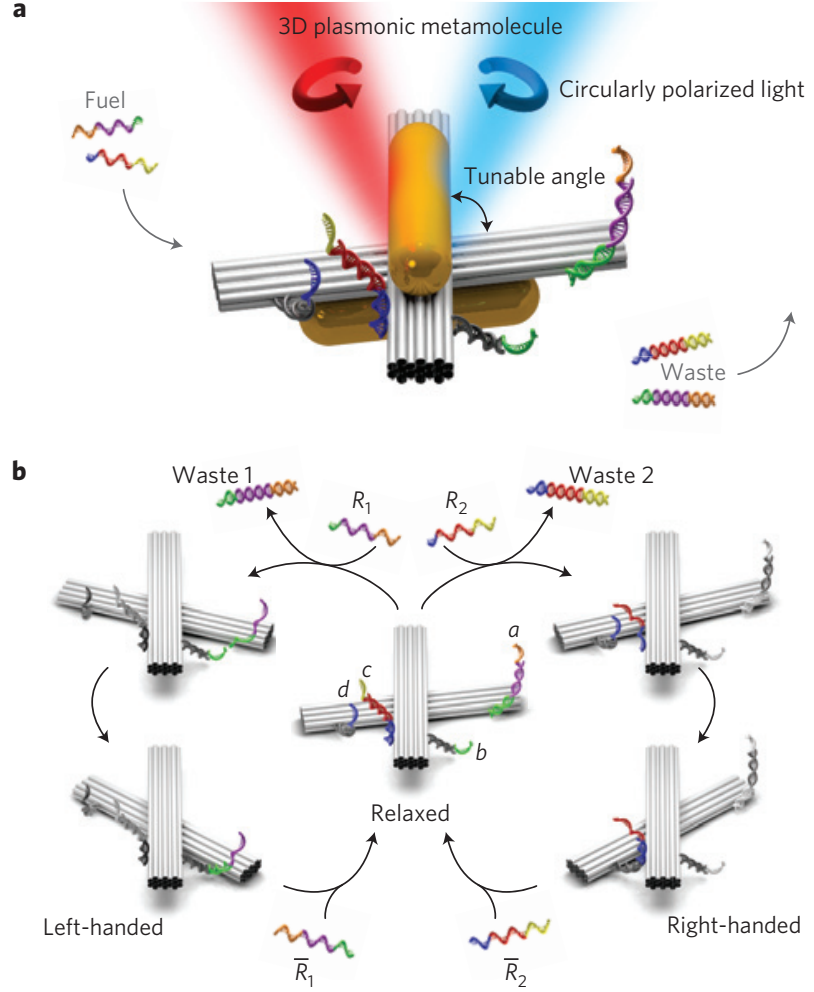

Figure 1 | Reconfigurable 3D plasmonic metamolecules. a, Schematic diagram. Two gold nanorods (AuNRs) are hosted on a switchable DNA origami template consisting of two connected bundles, which subtends a tunable angle. The relative angle between the AuNRs and therefore the handedness of the 3D chiral nanostructure can be actively controlled by two DNA locks, which are extended from the sides of the DNA origami template. Specifically designed DNA strands work as fuel to drive the plasmonic nanostructure to desired states with distinct 3D conformations by altering the relative angle between the two DNA bundles and hence the AuNRs. Unreactive waste is produced during a cycle. The red and blue beams indicate the incident left-and right-handed circularly polarized light, respectively. $\mathbf{b}$, Switching mechanism. The four arms of the two DNA locks are labelled $a, b, c$ and $d$. Through toehold-mediated strand displacement reactions, the plasmonic nanostructure can be driven to either the left- or right-handed state by adding removal strands $R_{1}$ or $R_{2}$ and back to its relaxed state by adding return strands $\bar{R}_{1}$ or $\bar{R}_{2}$.

By adding specifically designed DNA fuel strands, the 3D plasmonic metamolecules can be switched between different conformational states, which are characterized by distinct CD spectra.

\footnotetext{
${ }^{1}$ Max Planck Institute for Intelligent Systems, Heisenbergstrasse 3, D-70569 Stuttgart, Germany, ${ }^{2}$ Fakultät für Physik and Center for Nanoscience, Ludwig-Maximilians-Universität, Geschwister-Scholl-Platz 1, 80539 München, Germany, ${ }^{3}$ Department of Physics and Astronomy, Ohio University, Athens, Ohio 45701, USA. †Present address: Clarendon Laboratory, Department of Physics, University of Oxford, Parks Road, Oxford OX1 3PU, UK. *e-mail: kuzyk@is.mpg.de; laura.liu@is.mpg.de
} 

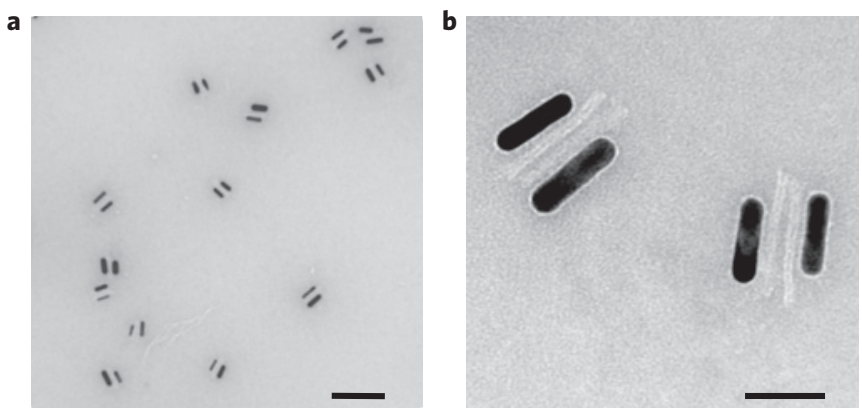

Figure 2 | TEM images of the plasmonic metamolecules in the right-handed state. a, Wide-field view. b, Magnified view. Two AuNRs $(38 \mathrm{~nm} \times 10 \mathrm{~nm})$ are accurately attached on one DNA origami template, which consists of two 14 -helix bundles $(80 \mathrm{~nm} \times 16 \mathrm{~nm} \times 8 \mathrm{~nm})$.

The structures tend to lie flat on the TEM grid. Scale bars, $200 \mathrm{~nm}$ (a); $50 \mathrm{~nm}(\mathbf{b})$

The switching mechanism is illustrated in Fig. 1b. To highlight the DNA-regulated dynamic flow, AuNRs are omitted in this figure. Two DNA locks that are used to manipulate the configuration of the plasmonic metamolecule are extended from the sides of the DNA origami bundles. Each lock contains two DNA arms. In a relaxed state, both of the DNA locks are open as illustrated in the centre image in Fig. 1b. First, we elaborate the left cyclic process. The colour of the non-involved DNA lock is understated for a clear illustration. Arm $a$ possesses a double-stranded DNA (dsDNA) segment (green and violet) with a 10-nucleotide (nt)-long ssDNA toehold (orange). Arm $b$ possesses a dsDNA 20-base-pair segment (grey) with a 12-nt-long locking sequence (green; also see Supplementary Fig. 4). When removal strand $1\left(R_{1}\right)$ is added, dissociation of the DNA strand with the toehold from arm $a$ is triggered through a branch migration process ${ }^{23}$, producing unreactive waste 1 . The locking strand (green) in arm $a$ is spontaneously available for hybridization with its complementary strand (green) in arm $b$. Subsequently, the two DNA origami bundles are joined together and the system is directed to a left-handed state. It is noteworthy that the stiff dsDNA segment (grey) in arm $b$ is particularly employed here to define a rigid angle between the two DNA origami bundles and therefore establish a stable state of defined handedness after the structural transition. With the addition of return strand $1\left(\bar{R}_{1}\right)$, the DNA lock is pulled open through a second DNA branch migration process mediated by a 12 -nt-long toehold sequence (violet). Then, the system returns to its relaxed state. In short, the DNA lock can be closed and opened through sequential hybridization and dehybridization of its two arms, giving rise to controlled switching of the system between different states. Following a similar route, the system can be reconfigured between its relaxed and right-handed state by successive addition of removal strand $2\left(R_{2}\right)$ and return strand $2\left(\bar{R}_{2}\right)$ as illustrated in the right part of Fig. 1b (see also Supplementary Fig. 5). The designated angle between the two DNA origami bundles is approximately $50^{\circ}$ for both the left- and righthanded states as this angle is correlated to the strongest CD for our system (Supplementary Fig. 20).

Transmission electron microscopy (TEM) was carried out to examine the as-fabricated nanostructures. In Fig. 2, locked plasmonic nanostructures in the right-handed state are shown. TEM images of other configurations can be found in Supplementary Note 4 . Figure $2 \mathrm{a}, \mathrm{b}$ presents an overview and a magnified view
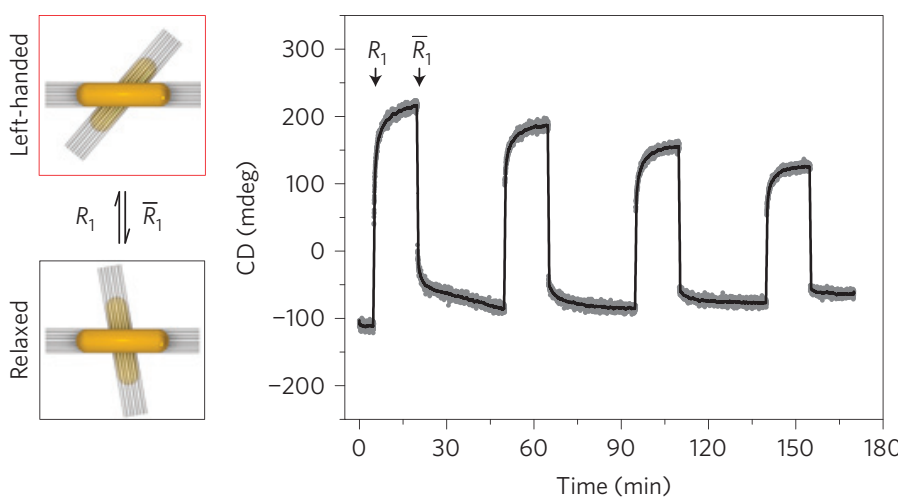

c
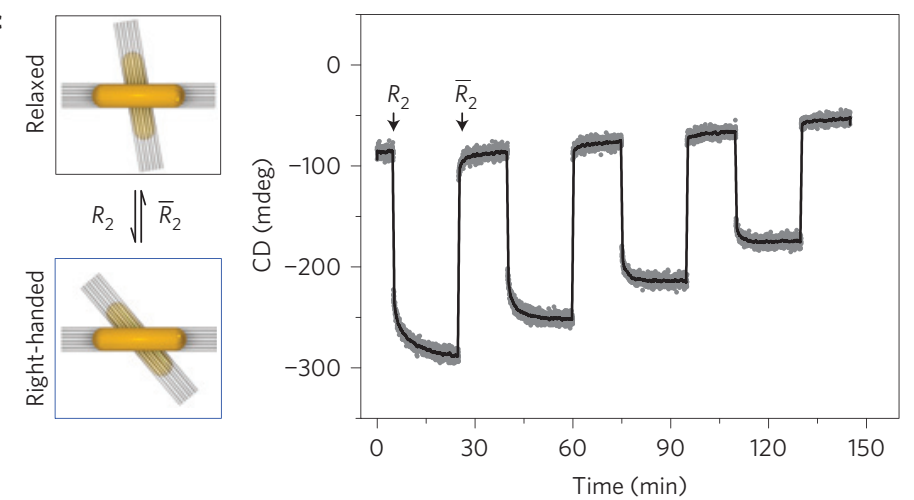

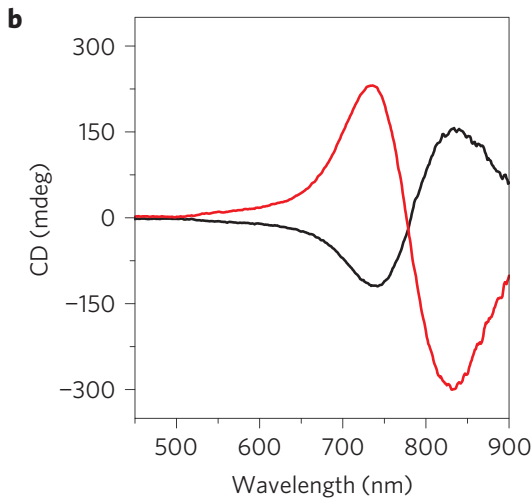

d

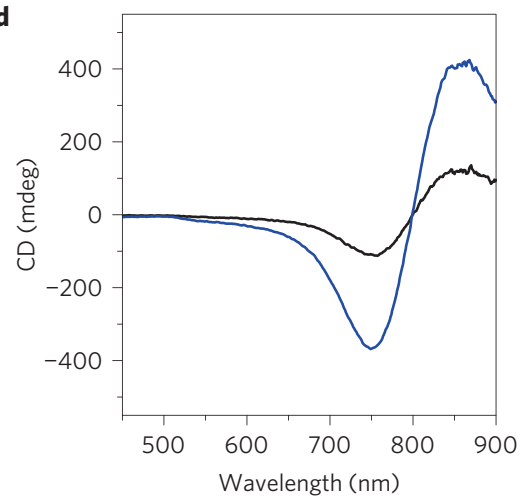

Figure 3 | Cycling the 3D plasmonic metamolecules between two states. a-d, The plasmonic metamolecules may be switched between the left-handed and relaxed states by adding $R_{1}$ and $\bar{R}_{1}$ in sequence $(\mathbf{a}, \mathbf{b})$ or between the right-handed and relaxed states by adding $R_{2}$ and $\bar{R}_{2}$ in sequence (c,d). The CD signal was monitored over time at a fixed wavelength of $725 \mathrm{~nm}$. b, CD spectra measured in the first cycle of $\mathbf{a}$. The red and black curves correspond to the CD spectra of the left-handed and relaxed states, respectively. $\mathbf{d}$, CD spectra measured in the first cycle of $\mathbf{c}$. The blue and black curves correspond to the CD spectra of the right-handed and relaxed states, respectively. The right-handed state exhibits much larger CD response than the relaxed state. The relaxed state exhibits a slight right-handed preference. 
a

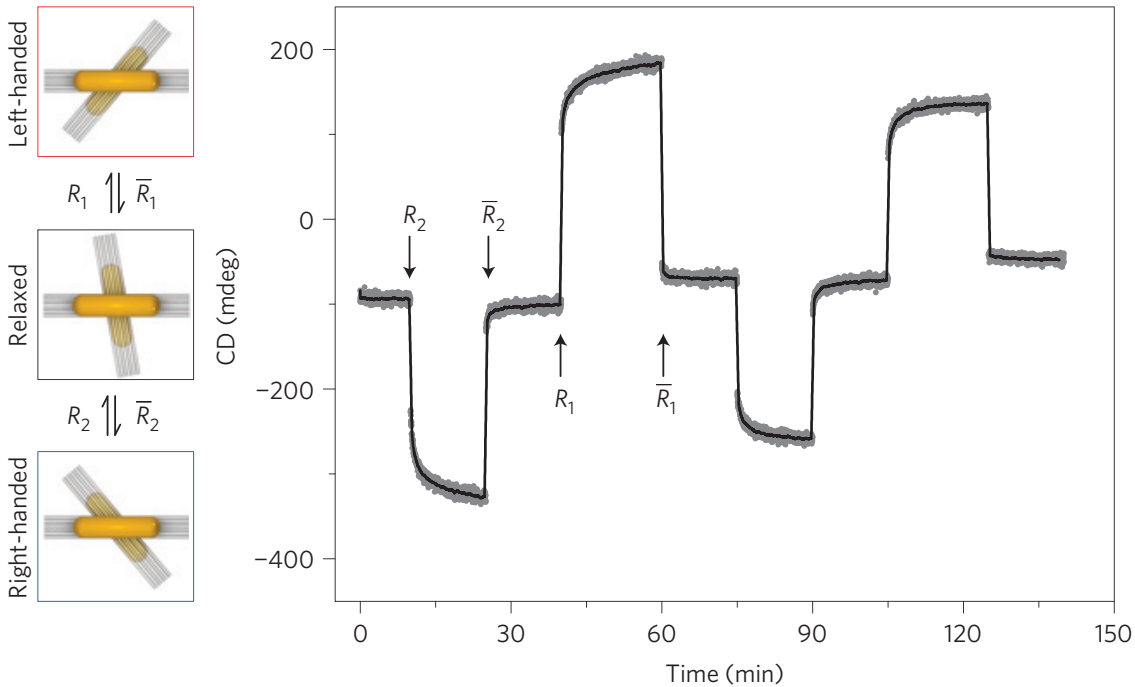

b

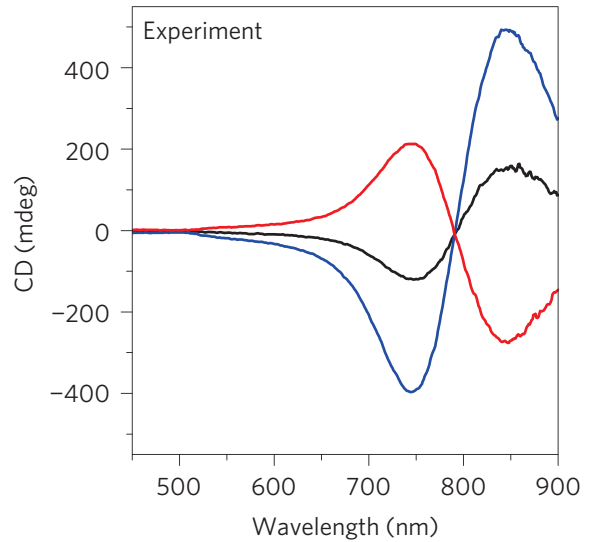

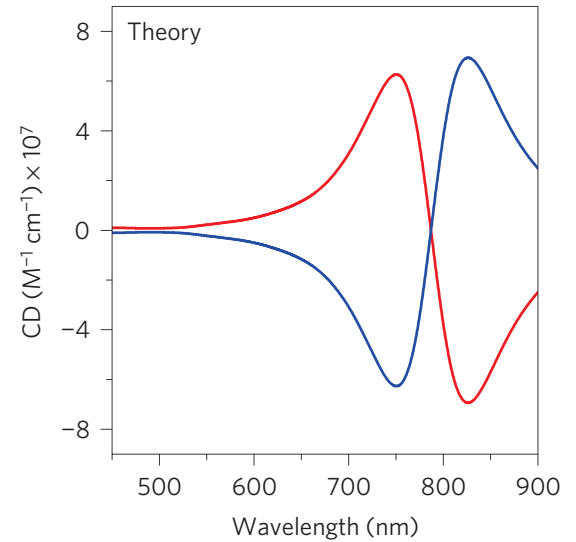

Figure 4 | Cycling the 3D plasmonic metamolecules between three states. a, The plasmonic metamolecules can be driven to either the left- or right-handed state by adding $R_{1}$ or $R_{2}$ and back to the relaxed state by adding $\bar{R}_{1}$ or $\bar{R}_{2}$. The CD signal was monitored over time at a fixed wavelength of $725 \mathrm{~nm}$. b. CD spectra measured in the first cycle of $\mathbf{a}$. The red, black and blue curves correspond to the CD spectra of the left-handed, relaxed and right-handed states, respectively. The right-handed state exhibits much larger CD response than the relaxed state. $\mathbf{c}$, Theoretical calculations based on a discrete dipole approximation method for the left- (red) and right-handed (blue) states. The angle between the two AuNRs was $50.7^{\circ}$ for both the left- and right-handed states in the calculations. The agreement between experiment and theory is qualitatively good.

of the right-handed structures, respectively. The structures exhibit certain deformation due to the drying process on the TEM grid. The DNA templates and AuNRs show adhesion preference to the grid surface. As a result, the crossed configuration in the right-handed state is difficult to visualize from these electron micrographs. Yet, it is evident from Fig. 2 that two AuNRs are accurately attached on one DNA origami template in each individual structure, demonstrating a successful assembly. Overall, we have achieved an assembly yield of the AuNR dimers as high as $90 \%$. This is also reflected in the strong CD response measured in the experiment, which will be shown next.

In situ CD spectra of the samples were measured using a J-815 CD spectrometer (Jasco). For the time-scan measurements, the CD signal was monitored over time at a fixed wavelength of $725 \mathrm{~nm}$. To ensure the stability and reliable functionalities of the DNA assemblies, all of the measurements were carried out at room temperature and $\mathrm{pH}$ 8.0. We first demonstrate that our plasmonic metamolecules can be cyclically reconfigured between the relaxed and a defined-handedness state. Figure 3a shows the optical trajectory of the system following a left-locking loop (see the left part of Fig. 1b). Switching between two distinct states can be clearly identified in Fig. 3a, where the CD signal successively alternates between negative and positive values. To achieve efficient transitions, the molar ratio of the metamolecules and the respective operating (removal or return) strands was kept to be $1: 140 \times N$, where $N$ is the cycle number (Supplementary Note 5). On adding $R_{1}$, the system shows rapid increase of the CD signal from negative to positive values. The transition takes approximately $15 \mathrm{~min}$ to complete. When $\bar{R}_{1}$ is added, the $\mathrm{CD}$ signal drops to negative as the system is reconfigured back to its relaxed state. The overall decrease in $\mathrm{CD}$ response during cycling is probably due to unintended interactions or secondary structure formation, which inhibit the restoration of $\bar{R}_{1}$ on $\operatorname{arm} a$. It is also partially due to dilution effects resulting from successive addition of removal and return strands. The decrease in performance ${ }^{24}$ for the left-handed route is $11.5 \%$ (Supplementary Note 5).

To provide a more complete picture, Fig. $3 \mathrm{~b}$ shows the corresponding CD spectra measured in the relaxed and lefthanded states in the first cycle. The CD spectrum of the lefthanded state in red exhibits a characteristic peak-to-dip line shape. Remarkably, the measured CD signal is as large as $250 \mathrm{mdeg}$ at a sample concentration of only $0.5 \mathrm{nM}$ (Supplementary Note 5). Such strong and distinct spectral response enables highly sensitive spectroscopy, which is the basis for optical monitoring of structural dynamics as well as ultrasensitive chiral sensing ${ }^{25}$. The relaxed state is characterized by a black curve, showing a dip-to-peak line shape. It implies that the right-handed crossed configuration is more 
favourable when both locks are inactive. This is probably due to a slight structural asymmetry introduced by the signature piece on the origami (Supplementary Fig. 1). As a control experiment, we also designed an achiral plasmonic structure, in which only one AuNR is assembled on the DNA origami template. The CD spectrum of the achiral sample exhibits negligible CD features in the whole wavelength range of interest (Supplementary Fig. 13). This elucidates that the pronounced CD response of the plasmonic system in the locked state originates from the strong plasmonic coupling between the crossed AuNRs.

Figure $3 c$ shows the evolution of the system following a rightlocking route (see the right part of Fig. 1b). Similarly to the process in the left-locking loop, on addition of $R_{2}$ the system departs rapidly towards more negative $C D$ values. As represented by the blue curve in Fig. $3 \mathrm{~d}$, the $\mathrm{CD}$ spectrum of the righthanded state in the first cycle exhibits a dip-to-peak line shape. The CD amplitude is much more pronounced than that measured in the relaxed state (see the black curve). A notable CD minimum of $-300 \mathrm{mdeg}$ is achieved. Subsequently, addition of $\bar{R}_{2}$ directs the system back to its relaxed state. The cycle continues with alternative addition of the removal and return strands. The decrease in performance for the right-handed route is about $10.4 \%$. When the two DNA locks work in sequence, the plasmonic system can be reconfigured between three distinct states: relaxed, lefthanded, and right-handed. The operation of the three-state cycle is shown in Fig. 4a and Supplementary Fig. 16. In brief, the system can be driven to either the left- or right-handed state by adding $R_{1}$ or $R_{2}$ and back to its relaxed state by adding $\bar{R}_{1}$ or $\bar{R}_{2}$. Figure $4 \mathrm{~b}$ presents the CD spectra measured in these three states in the first cycle. The CD spectra of the left- and right-handed states are characterized by the red and blue curves, respectively. They exhibit bisignated spectral bands with opposite chirality. The $\mathrm{CD}$ spectrum of the relaxed state in black shows right-handed preference, yet with much smaller amplitude. This also results in the CD response of the right-handed state stronger than that of the left-handed state. To provide deeper insight, theoretical calculations based on a discrete dipole approximation method ${ }^{26}$ were carried out (Fig. 4c and Supplementary Note 6). The CD spectra were calculated as difference in extinction for the left- and righthanded circularly polarized light. The assembled nanostructures were randomly dispersed in solution and therefore averaging over different orientations was performed ${ }^{27}$ (Supplementary Note 6). To account for the inhomogeneous spectral broadening resulting from the polydispersity of the AuNRs, the dielectric function of $\mathrm{Au}$ (ref. 28) was modified by including an extra damping coefficient (Supplementary Note 6). Overall, the agreement between experiment and theory is good. In the calculations, the right-handed preference in the relaxed state was not included. Therefore, the CD spectra of the left- and right-handed states show the same spectral profile with opposite chirality.

The concept of combining the know-how in plasmonics and DNA nanotechnology will stimulate a plethora of new research areas and real-life applications. The versatility of plasmonics may advance the development of smart probes for biochemistry and life sciences ${ }^{29,30}$. Such probes could allow for in situ monitoring of macromolecular and biological processes as well as dynamic transitions optically. For example, a single bio-entity that binds to the DNA arms and its subsequent reactions with other chemical compounds could trigger a series of conformational changes of the plasmonic metamolecule. With a combination of single-particle dark-field microscopy and CD spectroscopy ${ }^{22}$, the conformation changes of the plasmonic molecule arising from single bio-entity binding could be optically detected in real time. In addition, the unprecedented level of spatial and temporal control offered by DNA nanotechnology may advance the development of smart nanomaterials for plasmonics.
For example, a metamaterial composed of reconfigurable $3 \mathrm{D}$ plasmonic chiral metamolecules may lead to an optical super fluid, which could be actively switched between positive and negative refractive indices ${ }^{31,32}$.

Received 21 January 2014; accepted 9 June 2014; published online 6 July 2014

\section{References}

1. Driscoll, T. et al. Memory metamaterials. Science 325, 1518-1521 (2009)

2. Ou, J-Y., Plum, E., Zhang, J. \& Zheludev, N. I. An electromechanically reconfigurable plasmonic metamaterial operating in the near-infrared. Nature Nanotech. 8, 252-255 (2013).

3. MacDonald, K. F., Sámson, Z. L., Stockman, M. I. \& Zheludev, N. I. Ultrafast active plasmonics. Nature Photon. 3, 55-58 (2009).

4. Zhang, S. et al. Photoinduced handedness switching in terahertz chiral metamolecules. Nature Commun. 3, 942 (2012).

5. Zheng, Y. B. et al. Active molecular plasmonics: Controlling plasmon resonances with molecular switches. Nano Lett. 9, 819-825 (2009).

6. Seeman, N. C. DNA in a material world. Nature 421, 427-431 (2003).

7. Rothemund, P. W. K. Folding DNA to create nanoscale shapes and patterns. Nature 440, 297-302 (2006).

8. Krishnan, Y. \& Simmel, F. C. Nucleic acid based molecular devices. Angew. Chem. Int. Ed. 50, 3124-3156 (2011).

9. Yurke, B., Turberfield, A. J., Mills, A. P., Simmel, F. C. \& Neumann, J. L. A DNA-fuelled molecular machine made of DNA. Nature 406, 605-608 (2000).

10. Douglas, S. M. et al. Self-assembly of DNA into nanoscale three-dimensional shapes. Nature 459, 414-418 (2009).

11. Andersen, E. S. et al. Self-assembly of a nanoscale DNA box with a controllable lid. Nature 459, 73-76 (2009).

12. Ke, Y., Ong, L. L., Shih, W. M. \& Yin, P. Three-dimensional structures self-assembled from DNA bricks. Science 338, 1177-1183 (2012).

13. Castro, C. E. et al. A primer to scaffolded DNA origami. Nature Methods $\mathbf{8}$, 221-229 (2011).

14. Kuzyk, A. et al. DNA-based self-assembly of chiral plasmonic nanostructures with tailored optical response. Nature 483, 311-314 (2012).

15. Fasman, G. D. Circular Dichroism and the Conformational Analysis of Biomolecules (Plenum, 1996).

16. Wang, H., Brandl, D. W., Nordlander, P. \& Halas, N. J. Plasmonic nanostructures: Artificial molecules. Acc. Chem. Res. 40, 53-62 (2007)

17. Holliday, R. A mechanism for gene conversion in fungi. Genet. Res. 5, 282-304 (1964)

18. Halas, N. J., Lal, S., Chang, W-S., Link, S. \& Nordlander, P. Plasmons in strongly coupled metallic nanostructures. Chem. Rev. 111, 3913-3961 (2011).

19. Auguié, B., Alonso-Gómez, J. L., Guerrero-Martínez, A. \& Liz-Marzán, L. M. Fingers crossed: Optical activity of a chiral dimer of plasmonic nanorods. J. Phys. Chem. Lett. 2, 846-851 (2011).

20. Lan, X. et al. Bifacial DNA origami-directed discrete, three-dimensional, anisotropic plasmonic nanoarchitectures with tailored optical chirality. J. Am. Chem. Soc. 135, 11441-11444 (2013).

21. Zhao, Y., Belkin, M. A. \& Alù, A. Twisted optical metamaterials for planarized ultrathin broadband circular polarizers. Nature Commun. 3, 870 (2012).

22. Ma, W. et al. Chiral plasmonics of self-assembled nanorod dimers. Sci. Rep. 3, 1934 (2013).

23. Zhang, D. Y. \& Seelig, G. Dynamic DNA nanotechnology using strand-displacement reactions. Nature Chem. 3, 103-113 (2011)

24. Graugnard, E. et al. DNA-controlled excitonic switches. Nano Lett. 12, 2117 (2012).

25. Ma, W. et al. Attomolar DNA detection with chiral nanorod assemblies. Nature Commun. 4, 2689 (2013)

26. Draine, B. T. \& Flatau, P. J. Discrete-dipole approximation for scattering calculations. J. Opt. Soc. Am. 11, 1491-1499 (1994).

27. Fan, Z. \& Govorov, A. O. Plasmonic circular dichroism of chiral metal nanoparticle assemblies. Nano Lett. 10, 2580-2587 (2010).

28. Johnson, P. B. \& Christy, R. W. Optical constants of the noble metals. Phys. Rev. B 6, 4370-4379 (1972).

29. Liu, N., Hentschel, M., Weiss, T., Alivisatos, A. P. \& Giessen, H. Three-dimensional plasmon rulers. Science 332, 1407-1410 (2011).

30. Sönnichsen, C., Reinhard, B. M., Liphardt, J. \& Alivisatos, A. P. A molecular ruler based on plasmon coupling of single gold and silver nanoparticles. Nature Biotechnol. 23, 741-745 (2005).

31. Pendry, J. B. A chiral route to negative refraction. Science 306, 1353-1355 (2004).

32. Zhang, S. et al. Negative refractive index in chiral metamaterials. Phys. Rev. Lett. 102, 023901 (2009). 


\section{Acknowledgements}

We thank A. Jeltsch and R. Jurkowska for assistance with CD spectrometry. We thank M. Kelsch and H. Ries for assistance with TEM microscopy and DNA scaffold preparation, respectively. We acknowledge $\mathrm{S}$. Hein for material visualizations and F. Simmel for advice, respectively. TEM data were collected at the Stuttgart Center for Electron Microscopy (StEM). N.L. was supported by the Sofja Kovalevskaja Award from the Alexander von Humboldt-Foundation. A.K. was supported by a postdoctoral fellowship from the Alexander von Humboldt-Foundation. A.K. and N.L. were supported by a Marie Curie CIG Fellowship and the Grassroots Proposal M10330 from the Max Planck Institute for Intelligent Systems. T.L. and R.S. were supported by the Volkswagen Foundation and the DFG cluster of excellence NIM. H.Z. and A.O.G. were supported by the US Army Research Office under grant number W911NF-12-1-0407 and by Volkswagen Foundation (Germany). Use of the Computing Cluster at the Center for Nanoscale Materials was supported by the US Department of Energy, Office of Science, Office of Basic Energy Sciences, under Contract No. DE-AC02-06CH11357.

\section{Author contributions}

A.K., T.L. and N.L. conceived the experiments. A.K. and R.S. designed the DNA origami nanostructures. A.K. performed the experiments. H.Z. and A.O.G. carried out the theoretical calculations. A.K. and N.L. wrote the manuscript. All authors discussed the results, analysed the data and commented on the manuscript.

\section{Additional information}

Supplementary information is available in the online version of the paper. Reprints and permissions information is available online at www.nature.com/reprints.

Correspondence and requests for materials should be addressed to A.K. or N.L.

\section{Competing financial interests}

The authors declare no competing financial interests. 